\title{
NERVE ROOT COMPRESSION ASSOCIATED WITH NARROWING OF THE LUMBAR SPINAL CANAL
}

\author{
JOSEPH A. EPSTEIN, BERNARD S. EPSTEIN, \\ AND LEROY LAVINE \\ From the Department of Surgery, Division of Neurosurgery and Orthopedics, and \\ the Department of Radiology of the Long Island Jewish Hospital, \\ New Hyde Park, New York
}

During a study of low back pain and radiculitis, certain patients were observed who did not conform to the criteria of the classical compression syndromes caused by osteophytes or herniated discs (Epstein, 1960). Symptoms and signs of nerve root compression were far more severe than could be explained by the presence of relatively minor intrusions. Pre-operative myelograms disclosed single and often multiple defects of varying extent, some with complete block suggesting the presence of lesions more extensive than were encountered at operation. A significant common finding in these patients was the difficult operative exposure of the interspace caused by unusually prominent, bulbous articular facets and thickened, vertically orientated laminae. The spinal canal itself was shallow and exploration within its narrow confines was remarkably restricted. It became apparent that liberal unroofing of the spinal canal over the course of the nerve roots was as important for relief of symptoms as the excision of disc protrusions and spurs.

In reaching these conclusions, we were aided by previous experience in the management of a 15-year-old achondroplastic dwarf with compression of the conus and cauda equina by multiple lumbar intervertebral discs bulging into a congenitally narrowed spinal canal (Epstein and Malis, 1955). The deformities of the neural arches and the concomitant reduction in the width and depth of the spinal canal were similar in this patient and those of the present series. The protruding discs in the floor of the canal were left undisturbed, adequate relief being obtained by laminectomy alone. Gratifying restoration of function with relief of pain followed a decompressive laminectomy of the twelfth dorsal and of all the lumbar vertebrae, and there has been no recurrence of symptoms since January, 1954. Even a small herniated intervertebral disc in an achondroplastic dwarf may cause catastrophic trauma to the spinal cord and cauda equina because of the absence of available space for displacement of these structures within the narrow bony canal (Spillane, 1952; Schreiber and Rosenthal, 1952; Vogl and Osborne, 1949). A similar lack of space predisposes the patients in the present group to the untoward effects of neural compression by minimal intrusions.

\section{REVIEW OF THE LITERATURE}

Verbiest $(1954,1955)$ first recorded the observation that an incongruity between the capacity and the contents of the lumbar spinal canal may give rise to compression of the roots of the cauda equina. The relative predisposing stenosis described was not regarded as a manifestation of a general disturbance in the growth of the skeleton but possibly as an endogenously determined developmental problem. He described 13 men in the fourth and fifth decades of life in whom 'developmental narrowing' of the lumbar vertebral canal was regarded as the main cause of radicular symptoms. Symptoms resembling intermittent claudication appeared while walking, standing, or on exertion, tending to disappear with rest. Trauma was regarded as an aggravating factor in two patients. Low back and unilateral sciatic pain were the primary complaints. Loss of power in both lower limbs appeared on walking. Neurological alterations were not prominent and were considered disproportionate to the severe disability. Depressed patellar reflexes and absent ankle responses were noted in five cases. In five patients, there was anaesthesia over the sacral dermatomes or alterations involving the lower lumbar dermatomes. Acute symptoms of nerve root compression were precipitated by small disc protrusions and hypertrophic spurs. Myelography showed partial to complete block in all cases. The protein value of the spinal fluid was normal in one-half of the cases, slightly to moderately raised in the remainder. The resemblance of the anatomical findings in these patients to the vertebral structure of the achondroplastic dwarf was recognized at operation. The response to decompressive laminectomy, facetectomy, 
and removal of spurs and disc protrusions was gratifying, with 10 of 13 patients gaining complete relief of symptoms for as long as five years.

Schlesinger and Taveras (1953) have emphasized the significance of a narrow spinal canal in patients with herniated discs presenting an atypical picture of multiple root or cauda equina compression. Neoplasms were suspected because of subtotal or complete block on myelography and a raised spinal fluid protein level. It was found that the size of the disc protrusion was only secondary to the size of the spinal canal as the primary cause of root compression. Using the criteria of Elsberg and Dyke (1934) of the normal range of interpedicular measurements, they found values in these cases to fall within the extremely low end of the scale. Specific reference was made to the vertical laminar pattern and the exaggerated obliquity of the posterior articulations which add to the difficulty in surgical exposure. The disc extrusions were found to occur somewhat higher and well beneath the laminar arch and could easily have been missed or thought to have been dislocated upwards. These authors suggest that traumatic paralysis may well occur at the time of surgery because of a lack of appreciation of the structural peculiarities of these narrow canals.

Gill and White (1955) noted a marked reduction in the antero-posterior diameter of the spinal canal in patients with a transitional fifth lumbar vertebra. While a herniated disc may be found at the interspace above this abnormal vertebra, cases were encountered where only a mild bulge at this level could not satisfactorily explain symptoms of compression of the fifth lumbar nerve root, and often, the first sacral root. In the sacralized fifth lumbar vertebra, the diameter of the spinal canal was as small as the usual sacral canal. Early disc degeneration with bulging into this congenitally narrow canal would therefore produce evidence of root compression relatively easily. Laminotomy with removal of the disc provided relief of the neurological symptoms. However, persistent back and leg pain did not resolve until the operation was altered to include excision of the entire lamina of the transitional vertebra.

Mention must be made of the finding by Jefferson (1955) of localized enlargement of the spinal canal in the thoraco-lumbar region unassociated with neoplasm, cord anomaly, or arachnoidal cyst. The condition was considered to be essentially benign and without clinical significance. Hanraets (1959) also discusses the 'wide' and the 'flat-oval' lumbosacral spinal canal as developmental anomalies. The associated finding of a wide 'pseudo-cystic' dural sac in a number of such individuals with low back pain and radicular symptoms led him to imbricate the walls of the dural envelope with favourable results. He also discusses asymmetrical vertebral canals with disproportion between the left and right halves found with unilateral sacralization of the fifth lumbar vertebra.

Physicians treating disorders of the spine must be fully aware of the significance of variations in the internal configuration of the spinal canal if better results are to be obtained in the diagnosis and relief of syndromes caused by nerve root compression. For this reason, our experiences in the clinical, radiological, and surgical aspects of this problem have been reviewed.

\section{PRESENTATION OF CASES}

Twenty-nine patients with symptoms of nerve root compression causally related to narrowing of the lumbar spinal canal were studied. Seventeen were in the fifth and sixth decades of life. Five were in their thirties and five in their sixties. One patient was under 30 and one over 70 years of age. Fourteen were women. The most conspicuous symptom was pain. Complaints were initiated in 13 patients by falls or by lifting weights.

Stress or injury was not a predisposing factor in the remainder. Walking, postural changes, coughing, and straining were known to aggravate symptoms. No characteristic body habitus was observed. Occasional remissions were noted, but more often disability progressed despite bed rest, traction, and the use of various back supports. In nine, radicular pain was so intense that hysteria was suspected. Fourteen came to surgery within six months after the onset of symptoms. Six had been observed up to one year, eight for two to three years before being explored. The usual history was one of low back pain with radiation to one or both lower extremities. In five, the pain was present only in the hip and lower limb. The 10 most seriously disabled patients had bilateral lower extremity pain. Numbness and weakness were noted as pain increased but were not initial symptoms. Incontinence of urine and faeces was noted in a woman of 65 who presented with symptoms of bilateral root involvement. Impotence was present for eight months in another patient who did not have sphincter dysfunction.

Varying degrees of acute spasm of the lumbar musculature occurred in all. Severe pain on straight leg raising was present unilaterally in 17 patients, bilaterally in nine, and was not observed in three. In the group with bilateral restriction, six had been aware of symptoms on only one side. Depressed to absent deep tendon reflexes occurred in 22 patients. The ankle jerk was most seriously compromised, the patellar reflexes to a lesser extent. Sensory 
disturbances were detected in 20. The inner aspect of the foot and leg was involved in five patients (L-5 dermatome), the outer leg and foot in seven (S-1 dermatome). Multiple dermatomes were implicated in five additional patients and three showed severe defects in the saddle areas. Weakness was most evident in the distal musculature, including an almost complete bilateral foot drop in the most severely affected patient with the sphincter changes. Motor power was often difficult to test accurately because of the intense pain caused by manipulation and positional changes. The interspace localization could not be predetermined on clinical grounds with accuracy. The findings suggested more than a simple ruptured disc. Often neoplasm of the cauda equina or metastatic tumour was diagnosed.

Radiographs of the lumbo-sacral spine in 15 patients were within normal limits. Moderate osteophytic spurring and narrowing of discs was observed in 14. Considering the ages of the patients, no definite significance was attributed to these alterations. Employing the criteria of Elsberg and Dyke (1934), the interpedicular spaces were in the lower limits of normal in 10 patients and never below these levels. After reviewing the films in retrospect, it was apparent that certain changes could be observed often enough to be helpful. These included a relative flattening of the intervertebral foramina in the dorso-ventral diameter indicating a shortening of the pedicles and an increased bony density in the dorsal segments in lateral radiographs corresponding to the augmented thickness of the lamina. An antero-posterior diameter of the spinal canal in the lumbar region of less than $1.5 \mathrm{~cm}$. was regarded as suggestive of significant narrowing. These findings, either singly or in combination, were recognized in 18 of the 29 cases.

Lumbar puncture at the level of the third or fourth interspace was often difficult, and occasionally impossible, when narrowing of the canal was further complicated by spondylotic changes. This alone was sufficient to arouse the suspicion of a structural variation. The total protein level in the spinal fluid was normal with a slight elevation of 50 to $60 \mathrm{mg}$. per $100 \mathrm{ml}$. in 10 patients. Only one had a protein content of $120 \mathrm{mg}$. per $100 \mathrm{ml}$.

Myelography was most informative. Measurement of the depth of the column of oil in the lateral exposure disclosed narrowing of the canal varying from 0.8 to $1.5 \mathrm{~cm}$. In five cases, a defect in the dorsal aspect of the oil column was related to inward protrusion of the ligamentum flavum and thickened lamina. In the antero-posterior position, varying bilateral waist-like defects were noted, including partial to complete block of the canal.
The areas of constriction were mainly at the level of the lower three lumbar interspaces, the third and fourth predominating (Figs. 1, 2, 3, 4). These abnormalities closely resemble the alterations found in advanced spondylosis, the two not infrequently being allied (Epstein and Epstein, 1957; Epstein, 1960).

A lack of fillıng in the lateral recess indicates only that there is insufficient space for the oil to accumulate in this corner. The defect in itself would not indicate the actual degree of compression of the nerve root since often a small indentation is associated with severe trauma to the enclosed nerve root. These patients require exploration of multiple interspaces not only to expose the immediate pathological changes but also to prevent future difficulties.

Of special interest was the presence of complete obstruction of the spinal canal in five patients, and partial obstruction in seven others (Figs. 1 and 2). This occurred about equally at the third or fourth interspace in 11 cases and at the fifth interspace in one. Four of these showed multiple defects such as a complete or partial block at one level with varying bilateral defects at adjacent levels (Fig. 2). Initial interpretations included a completely extruded disc, arachnoiditis, and cauda equina tumour. Operation disclosed extreme narrowing of the spinal canal. Relatively small ridges or disc protrusions in these patients were sufficient to precipitate symptoms in the presence of a narrowed canal.

In six patients there were unilateral defects in the column of oil without appreciable obstruction. Similar bilateral defects were found in 11 patients with a primary unilateral compression syndrome (Fig. 3). The severity of pain on one side, however, often masked symptoms on the other. Objective evidence of contralateral signs in the presence of often symmetrical myelographic defects was an indication for bilateral decompression. The multiple myelographic defects may be confusing. They should not mistakenly be regarded as artifacts.

Operation was performed with the patient in the crouch position. The buttocks were supported on a ledge with the knees and hips flexed, care being taken to avoid vascular compression. The absence of pressure on the abdomen and chest helped to prevent problems of venous congestion and bleeding from the epidural venous plexus.

After incising the lumbodorsal fascia and separating the sacrospinalis muscle mass, the unusual characteristics of the posterior elements immediately become apparent (Fig. 4). The bulbous posterior articular facets approach the spinous processes. The thick laminae are foreshortened in their transverse diameters and overlap. An exaggerated vertical 

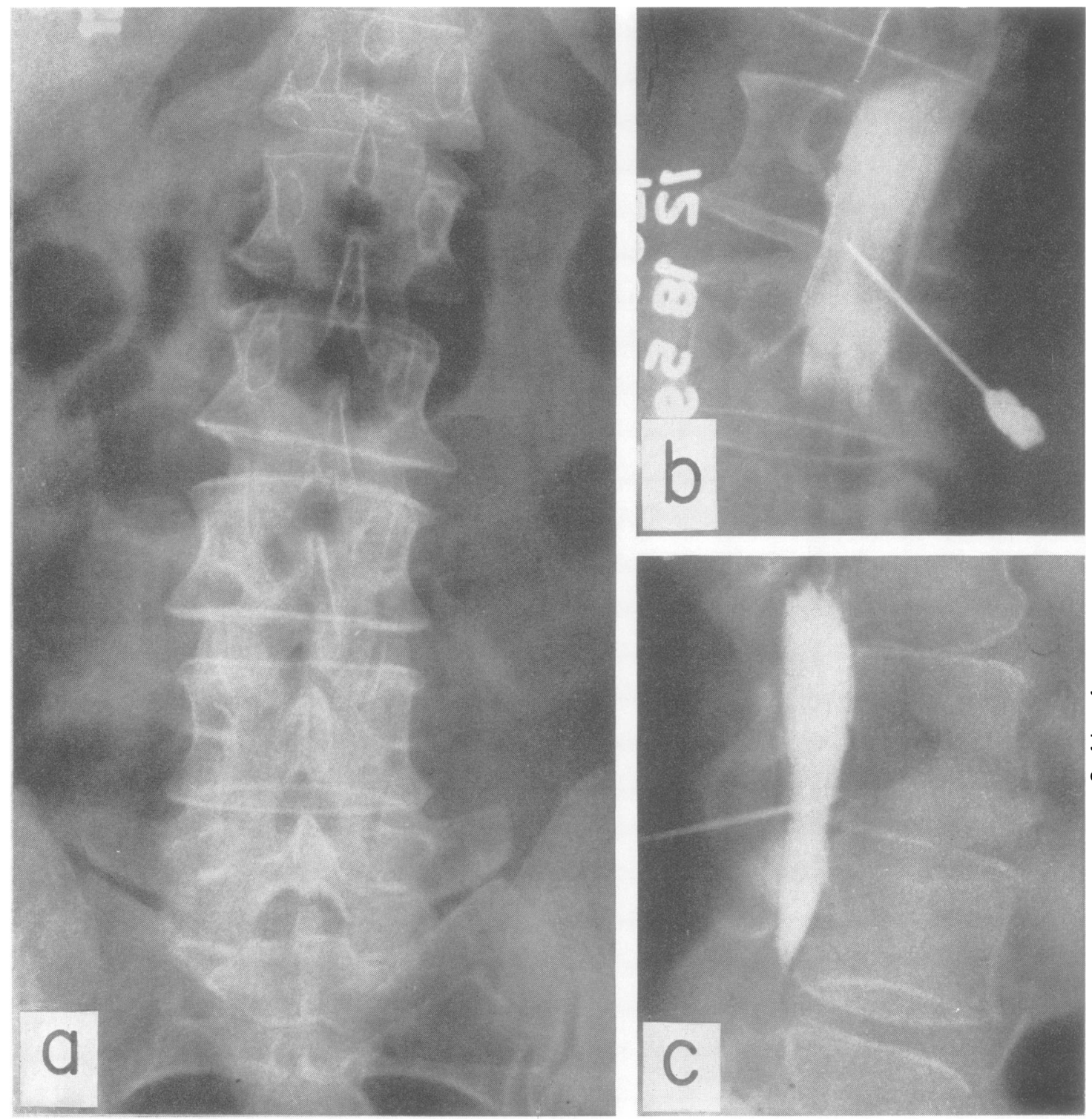

FIG. 1. These are films of a 72-year-old woman with intense left sciatic pain of six months' duration. Walking caused marked distress. The left ankle reflex was absent; there were no sensory changes.

a Anteroposterior view of the lumbar spine showing scoliosis to the right. The laminae at the fourth and fifth levels are thickened, the laminar angle acute at the third and fourth segments.

b A complete block is noted in the anteroposterior (slightly oblique) myelogram.

c The lateral study shows the typical pointed obstruction of the column of oil at the fourth interspace described by Verbiest $(1954,1955)$ as classical in patients with this disorder. At operation in December 1959 the canal was remarkably narrowed both in the dorso-ventral and lateral diameters. A slight bulging of the intervertebral disc had further compromised an already narrowed canal to the point of critical compression. Remarkable relief of pain followed laminectomy of the adjacent vertebrae and excision of the softened disc material. 

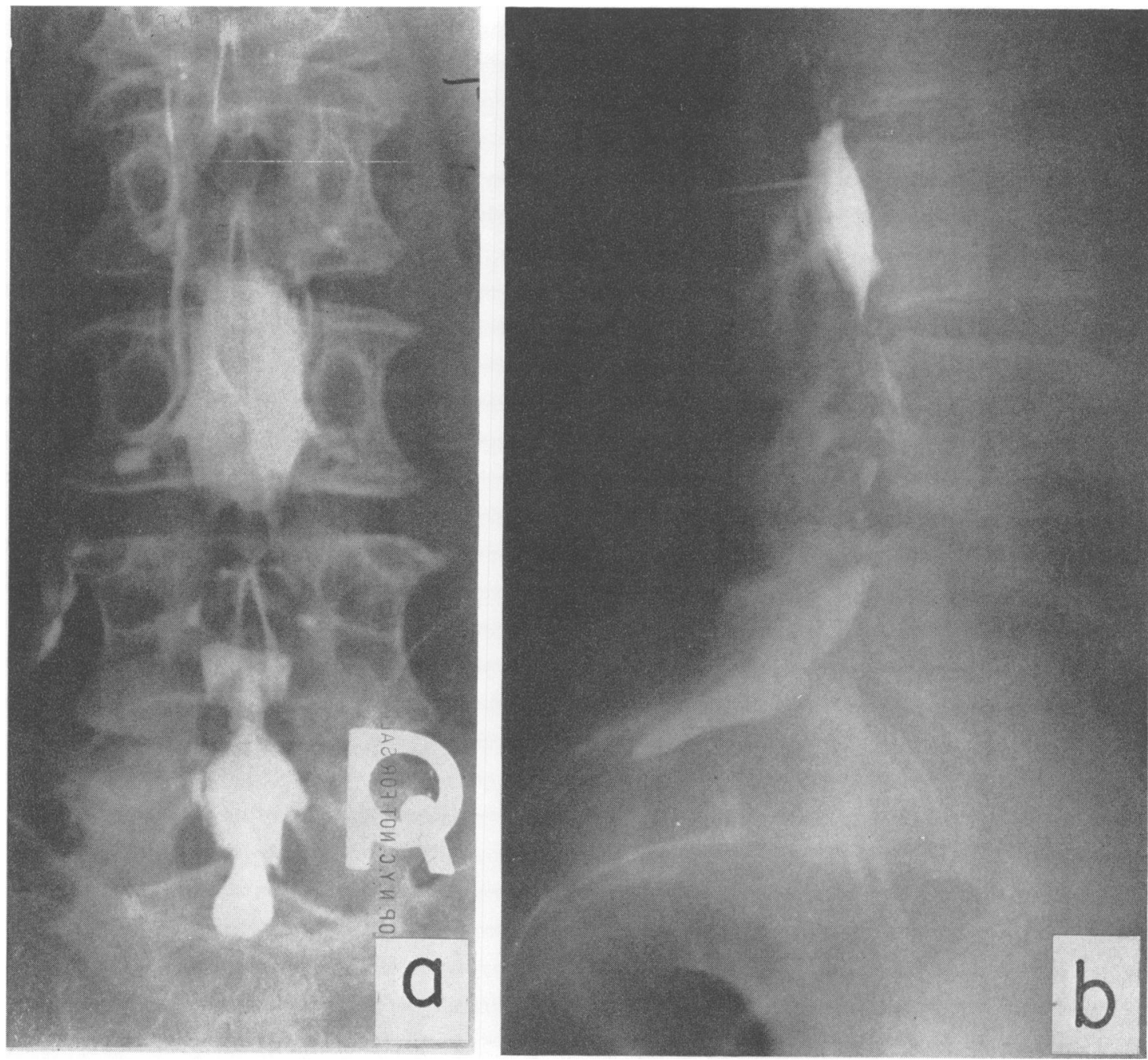

FIG. 2. This patient was a 67-year-old woman complaining of intractable left sciatic pain of three months'duration. The ankle reflex was absent and there were minimal sensory changes. The anteroposterior myelogram (a) shows a partial block at the third lumbar interspace with bilateral defects at the fourth and fifth levels. A characteristic pointed defect is seen in the lateral projection (b) at the level of partial block typical of compression of the dural sac by bothdorsal (lamina, yellow ligament) and ventral (disc) intrusion. The canal widens at the level of the fifth lumbar vertebra. A small extruded fragment of disc was removed from the fifth interspace on the left side. Total laminectomy of the third, fourth, and fifth vertebrae was performed with excision of bulging, degenerated discs at the other levels in May 1960. The result thus far has been gratifying. 


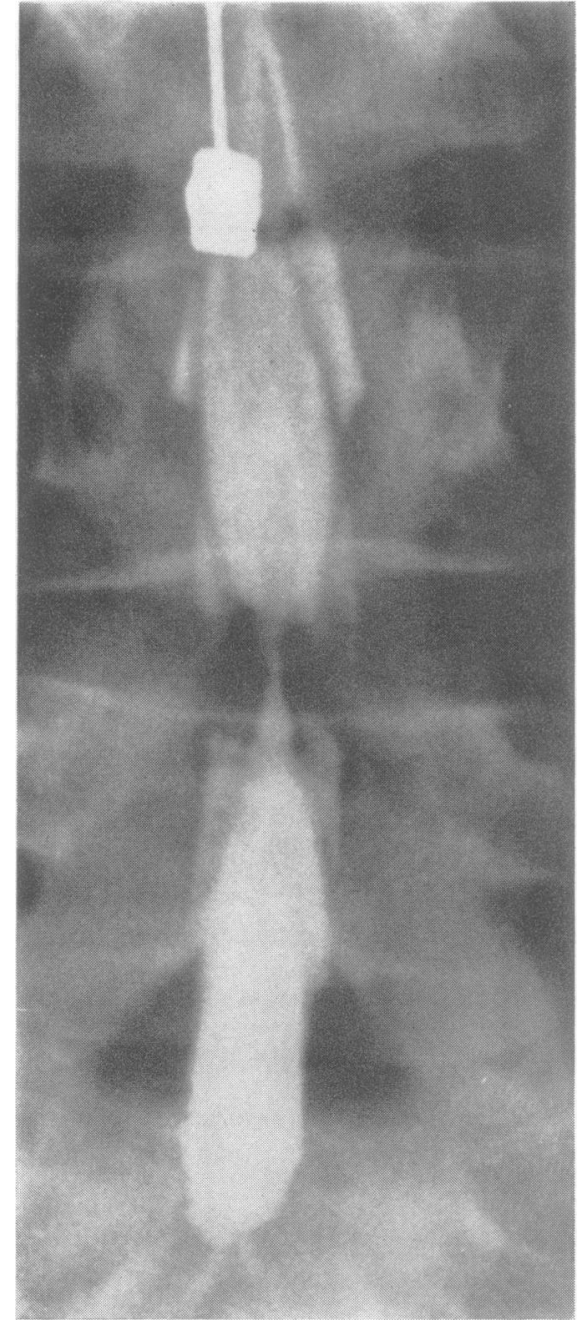

FIG. 3. Myelogram showing one of the common alterations, namely a bilateral waist-like defect at the L4 to L5 interspace.

This patient was a 44-year-old woman with low back pain and left sciatica for three months. The ankle reflex was absent and had a sensory deficit over the outer aspect of the calf and foot. Note that the side of the acute symptoms cannot be foretold by the appearance of myelogram alone and that the changes should not be disregarded as an artifact. The thread of Pantopaque connecting the divided column of oil lies in the deepest, mid-portion of the triangular canal. The defect only indicates the shallowness of the lateral recesses that cannot fill with oil and the amount of further acute compression can only be indicated by the clinical findings and at operation. The usual interlaminar laminotomy on the left with removal of a degenerated, bulging disc provided only temporary relief, and symptoms recurred bilaterally requiring subsequent laminectomy and foramenotomy at the fourth and fifth lumbar segments.

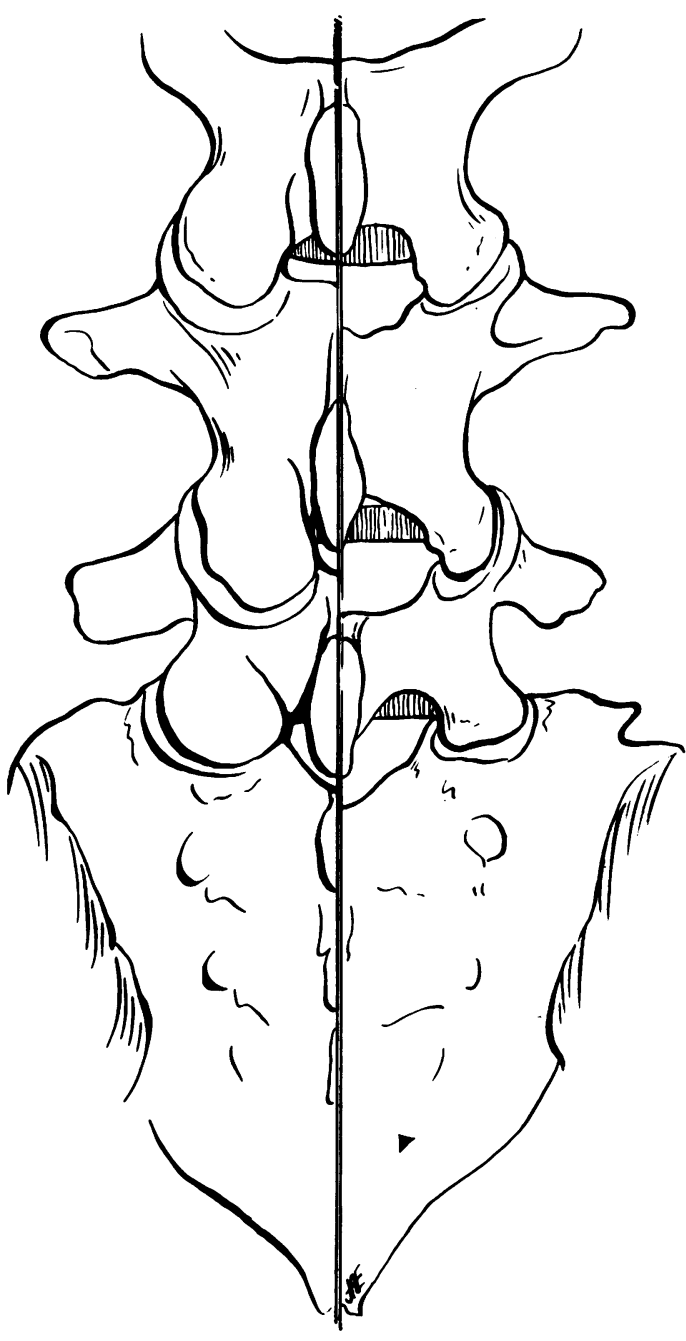

FIG. 4. Operative diagram showing the normal pattern of the articular processes and laminae on the right side, the unusual pattern found in patients with narrowing of the spinal canal on the left. Note particularly that the facets are unusually prominent and approach the midline; the laminae are thick with little space between them. The pattern may vary, the facets in certain patients being less prominent than illustrated. The laminar pattern, however, is more constant, with the overlapping of these structures presenting one of the major surgical difficulties in uncovering the canal. 


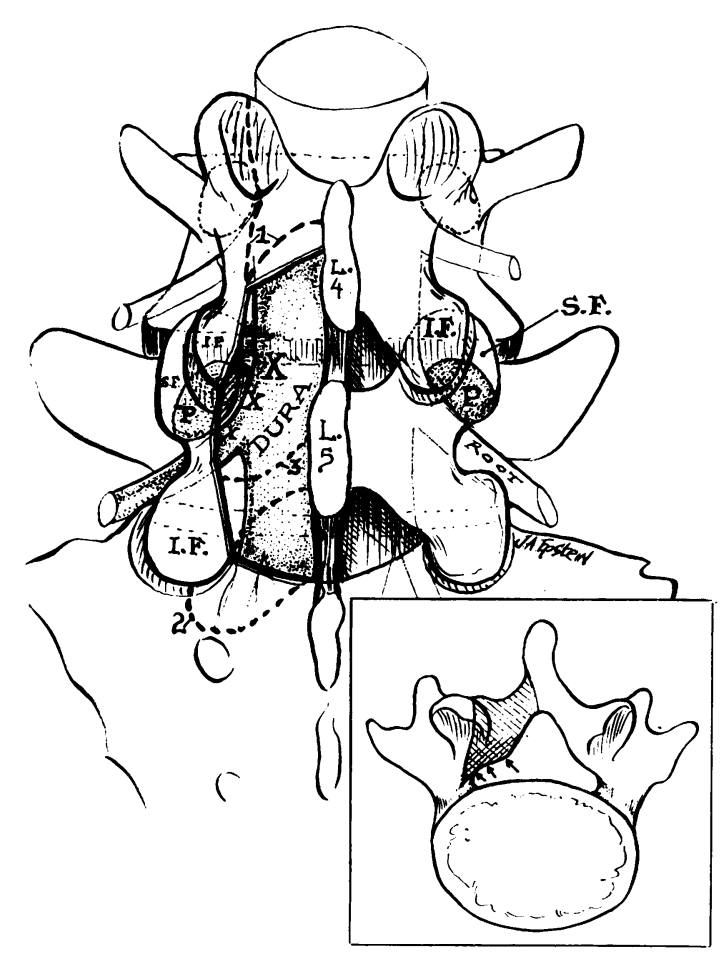

FIG. 5. Operative diagram indicating the extent of laminar removal (double line) on the left. The right side remains intact for purposes of orientation. Hemilaminectomy of the fifth lumbar vertebra has been performed, partial of the fourth with unroofing of the lateral recess and proximal portion of the foramen as the fifth lumbar nerve root crosses over the interspace and winds around the pedicle $(P)$ to its exit from the canal. The medial portion of the superior facet (S.F.) of the fifth lumbar vertebra forms the roof of the lateral recess in this critical area where major narrowing and compression by ventral spurs and discal protrusion occurs. The vital areas $X-X-X$ - must be clearly exposed. The most critical narrowing and compression occurs at the site marked by the largest $X$, at the superior lip of the vertebral body. The dotted lines $1-2-3$ indicate the extent of laminae removed that may be required for single or multiple lesions. The entire procedure may be performed bilaterally if indicated. The insert shows the shape of the spinal canal and the extent of vertical removal of lamina and facet needed to unroof the canal at the lateral recess and proximal portion of the intervertebral foramen (shaded area). Kerrison rongeurs with the cutting tip bevelled at $30^{\circ}$ to $40^{\circ}$ and with a thin lower lip facilitate removal of the roof of the lateral recess. obliquity of the laminae in the dorso-ventral plane makes it difficult to uncover and remove the ligamentum flavum and expose the canal. Little or no epidural fat is seen in the lateral recesses.

The alterations indicated occur in varying degrees of severity. The most profound changes are reflected in maximal narrowing of the spinal canal with a complete or partial block on the myelogram. Those less seriously involved show fewer apparent changes in the posterior articulations and lamina. All share the primary abnormality of clinical significance, namely, shallowness of the lateral recesses.

The critically limited reserve space for the nerve root makes it vulnerable to compression by a slight bulging of softened fibrocartilage, a small osteophyte or fragment of extruded disc. The entrapped root may be overlooked if the recess is not completely uncovered. Decompression is not considered complete until the root is unroofed as it passes over the annulus and margin of the vertebral body, around the pedicle and through the proximal portion of the foramen (Fig. 5). The yellow ligament is excised along with all significant intrusions of discal material and osteophytes. All available degenerated disc tissue is removed from the interspace. Portions of the articular facets are sacrificed only if necessary for exposure.

The dura was opened on two occasions because of a total block on myelography, each patient being suspected of harbouring a neoplasm. No intradural pathology was noted, the defect being caused by the extreme narrowness of the canal, further compromised by the slight bulging of the underlying degenerated disc.

Tiny extruded fragments of disc with an aggregate volume of approximately $0.5 \mathrm{c.cm}$. were found in seven patients. Bulging soft discs with varying degenerative changes were noted in the remainder. Osteophytes contributed to further constriction of the canal in 10 patients. Interlaminar laminotomy with removal of the apposing half of each adjacent lamina was the initial operative approach to the interspace in 10 patients. This was subsequently thought to be inadequate in six. Complete hemilaminectomy with excision of additional portions of the adjacent lamina was performed in four patients. Total laminectomy of one to three vertebrae was performed in 15 patients, the extent being determined by myelographic evidence of block and multiple defects.

The patients were kept flat on their backs for six to eight hours after operation and were then encouraged to turn from side to side and to assume any position of comfort. Walking was permitted by the third to fifth post-operative day. Sedentary work was allowed in four to six weeks, more arduous 
activity in two to three months. Spine fusion was not considered necessary even in those patients with facet injury.

Our patients have been followed from one to three and one-half years. Thirteen returned to normal activity without restriction or significant complaint and were considered as excellent results. An additional 10 had good results, leading productive lives although slightly incapacitated and not requiring specific therapy beyond restriction of arduous activity. Two fair results occurred in patients who still required supportive therapy with significant limitation of activity. The four patients with poor results remained disabled with little or no response. In view of the present criteria emphasizing exposure and decompression, these patients are considered to have been insufficiently explored. Two subsequently were re-operated upon with more extensive decompression, neurolysis, and excision of additional degenerated disc tissue. One responded adequately and had a well sustained result. The second had partial relief as pain and disability recurred, possibly related to exuberant scar formation. Emotional difficulties and psychological elements connected with claims for compensation further clouded the issue.

The most gratifying response was the rapid relief of pain after surgery. Those with a long history of disability responded more slowly, with occasional noteworthy exceptions, leading to the belief that the nerve roots could withstand considerable trauma without permanent alterations. In this category would be included the patient with double incontinence in whom both bowel and bladder control returned nine months after surgery. The patient with an eight-month history of impotence had fair relief of pain but no return of adequate sexual activity after two years.

\section{DISCUSSION}

During the course of this investigation we examined the lumbar vertebrae of 50 persons from the collection of the Department of Anthropology of the Museum of Natural History in New York City. In five specimens, moderate to severe dorsoventral narrowing of the spinal canal was observed resembling that discovered at operation in our patients. In five additional preparations lesser abnormalities were found. The deformity of greatest clinical interest in all specimens was evident in the shallowness of the lateral recesses, which were found to measure as little as 2 to $3 \mathrm{~mm}$. compared with normal values of 5 to $7 \mathrm{~mm}$. (Figs. 6 and 7). Osteophytes from the vertebral margins further contributed to the constriction in two cases. Projections from the posterior apophyseal articulations were noted in two additional cases. The ages varied from 43 to 87 years, the majority being in the fifth decade of life. All were males. Unfortunately, no clinical information was available. While this represents a pilot study of limited proportions, the observations support our clinical impressions.

Verbiest (1954, 1955) and Hanraets (1959) discovered little of diagnostic importance in the interpedicular measurements of Elsberg and Dyke (1934) in evaluating patients with narrow canals. At operation, using a specially designed instrument to measure the depth of the canal, Verbiest (1955) claimed to have detected significant changes. Huizinga, van der Heiden, and Vinken (1951) used a new standard of measurement of the 'normal' spinal canal obtained from the vertebrae of 100 Netherlanders free of spinal disease. Their values were significantly lower than those of Elsberg and Dyke (1934) leaving considerable confusion in regard to the importance of such tables. Unfortunately, the depth of the canal at the lateral angles where the major compressive effects occur is impossible to estimate in ordinary radiographs or on laminography and we have relied primarily on myelographic and operative findings in the management of these patients. Discography is regarded as useless in that it provides no information concerning the internal dimensions of the spinal canal and may do a grave injustice to these patients since actual disc pathology may be minimal.

Statistical surveys concerned with the effects of surgery for herniated discs are difficult to evaluate because of the absence of any reference to such congenital or developmental variations. In our series of over 400 operations for discogenic disorders it can be estimated that from 10 to $15 \%$ of patients will show some deviation from normal as reflected in narrowing of the lateral recesses. An appreciation of the significance of these changes may help to explain and possibly avert many of the poorly understood surgical failures in this field. The removal of herniated discs or spurs may be inadequate to decompress an imprisoned nerve root or ganglion. Forceful insertion of an exploring instrument into a narrowed canal may crush and irreparably damage the contained nerve roots. The occasional relief of root pain following a so-called 'negative exploration' may conceivably be attributed to the decompressive effects of unroofing a narrowed canal and foramen.

The surgical technique developed during this study is similar to that of Verbiest (1955) and Hanraets (1959). The available space within the spinal canal is increased by excision of lamina, yellow ligament, and varying portions of facets where 

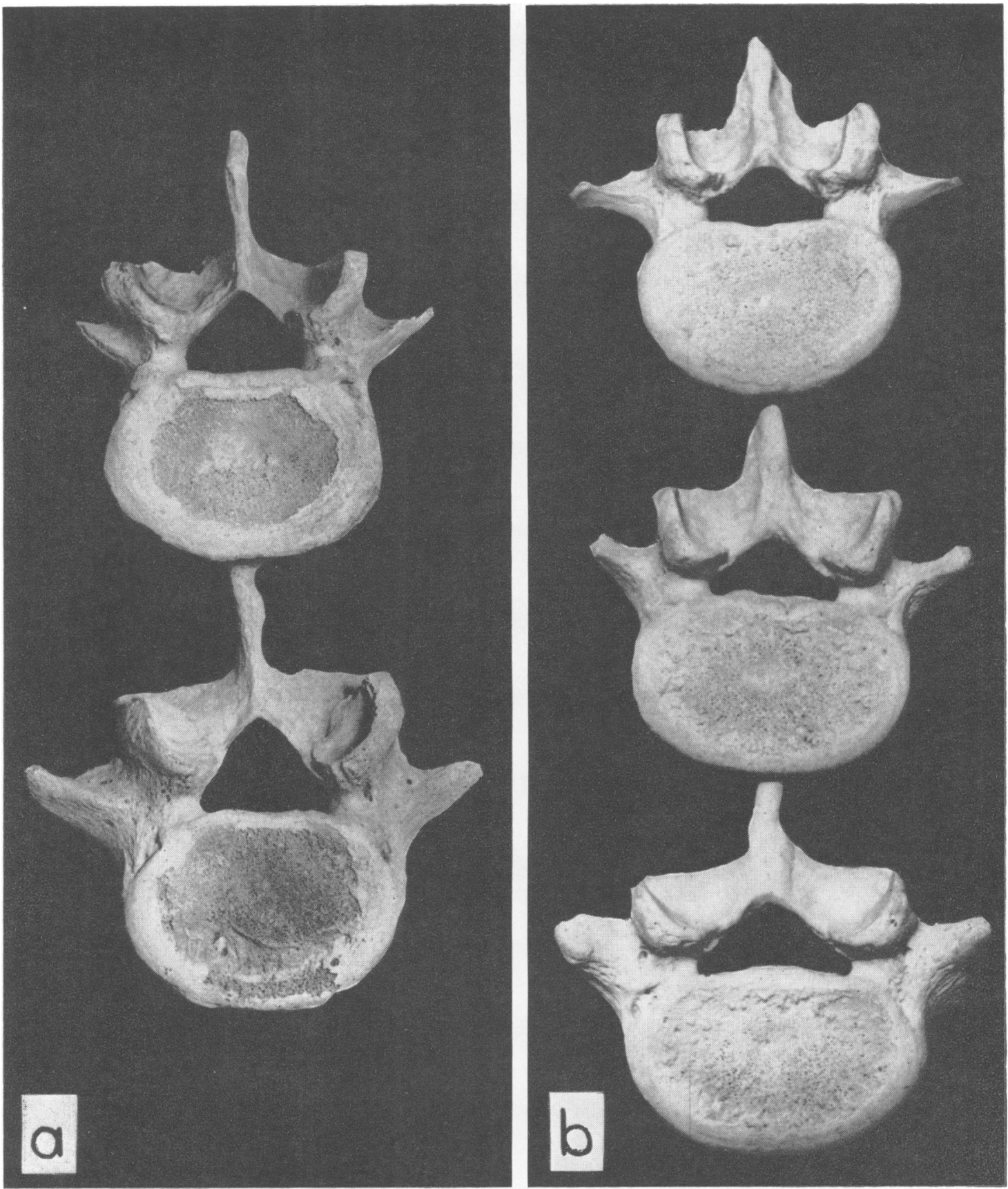

FIG. 6. The differences in the internal configuration of the spinal canal are evident in the two specimens. A normal fourth and fifth lumbar vertebra is seen in (a), taken from a 54-year-old man. The usual triangular shape with adequate lateral recesses is contrasted with the shape of the canal in the specimen (b) taken from a 58-year-old man where the triangular shape is flattened in the dorso-ventral diameter and the lateral recesses are narrow and restricted, extending beneath the shelving margins of the superior articular facets (cephalic view). 

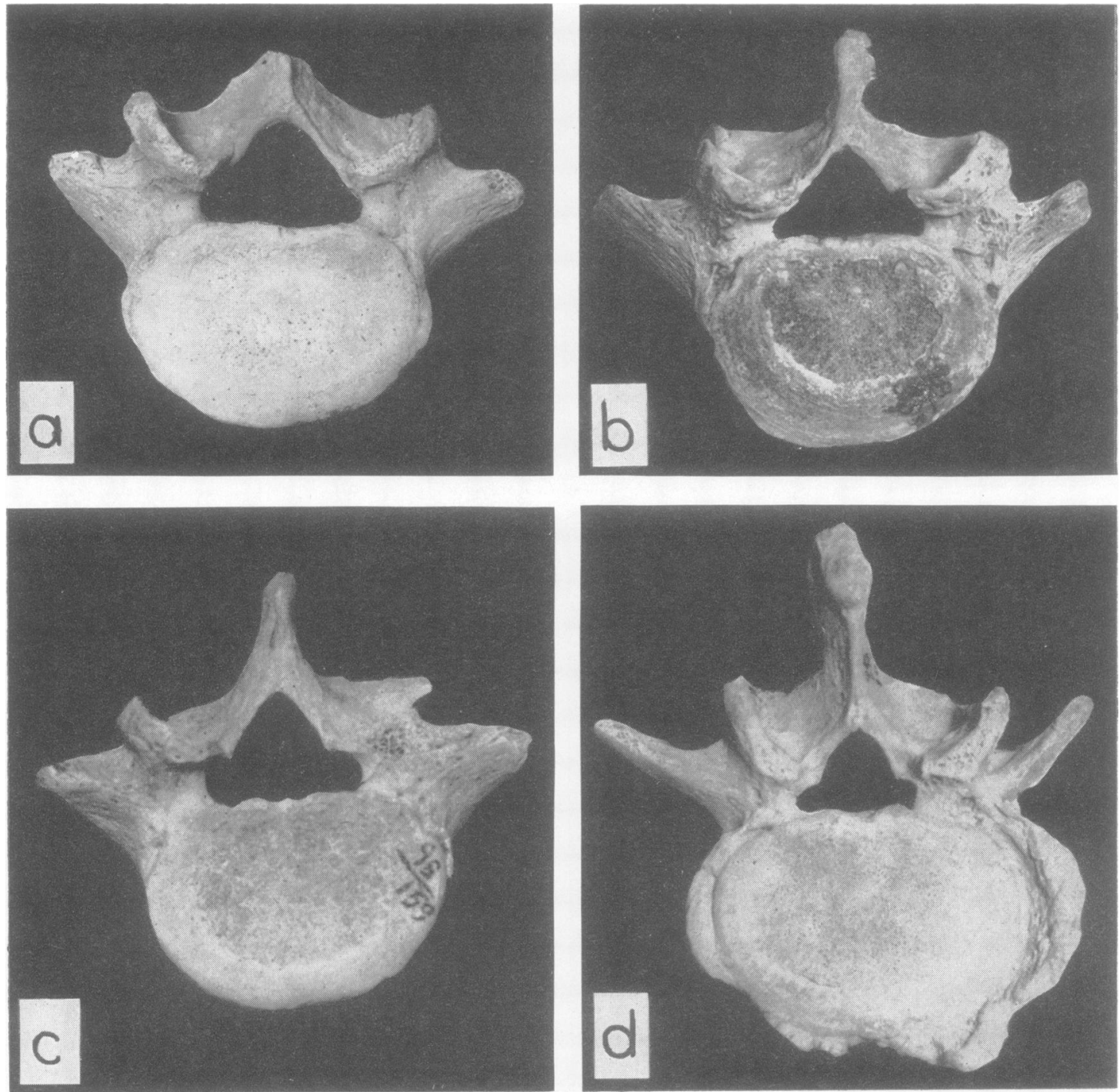

FIG. 7. The considerable variation in shape of the spinal canal is seen in these four specimens. A relatively normal pattern is illustrated in (a), the fifth vertebra from a 53-year-old man. Shallow lateral recesses are present in (b) and unusually notched lateral recesses are noted in (c), both representing the fifth lumbar segment from a 58-and a 43-year-old man. The fourth lumbar segment in (d) is from a 59-year-old man, illustrating an unusually small canal with an outline resembling that in (c). Note the greater vertical orientation of the superior facets.

needed for foraminal decompression. By undercutting the roof of the foramen with angulated Kerrison rongeurs this opening may be enlarged without sacrificing the superior articular process. In our patients, as in those of Hanraets (1959), no specific impairment of function could be attributed to excision of facets that did not exist before surgery. Munro (1956), after a considerable experience with
130 patients with herniated discs in whom facets were damaged or removed, states that 'the loss of a pair of intervertebral facets and their intervening joint space is of infinitely less importance than the continued root compression and inadequate exposure that would result from their retention or undamaged condition'.

In patients with lesser defects on myelography, 

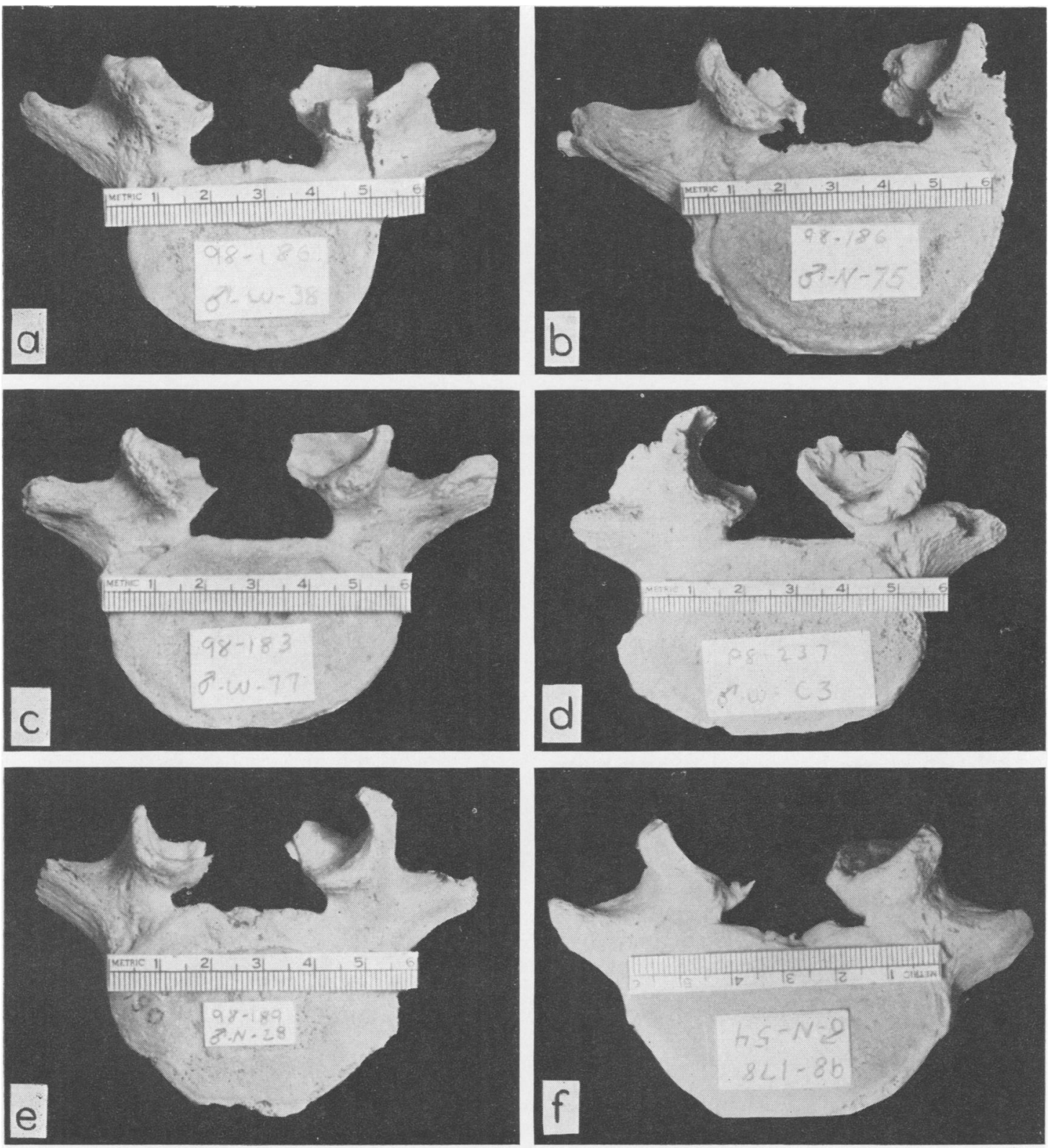

FIG. 8. The lumbar vertebrae illustrated are all from the fifth level and have had the laminae removed in the dissecting room. All demonstrate the variable narrowing of the lateral recesses and the frequent asymmetry that may have clinical significance. Relatively large recesses are seen in (a), from a man, 35 years of age. In (b), from a man of 75 years, the recess on the left extends far beneath the facet, an osteophyte extends downwards from the facet into the canal and a small ridge is seen in the floor of the canal and recess. The recess on the right appears adequate. In (c), from a 77-year-old man, a large recess is evident on the right, a shallow recess on the left. In (d), from a 63-year-old man, the entire canal is small, the recess extremely narrow and sharp on the right. In (e), from a 28-year-old man, the recess on the left is narrower than on the right. In (f), a more symmetrical type of narrowing is evident. Observe the varying angles of the surfaces of the facets and the effects of spondylosis and spondylitis on the shape of the canal. 
there may be a tendency at operation to ignore or incompletely to uncover the lateral recesses. This deficiency has led to re-exploration in two patients and is probably related to poor results in two others. One may conjecture that where minimal changes of questionable clinical significance exist a greater degree of narrowing may develop as a response to stress, longevity, and degenerative disorders. A congenital predisposition is suggested by the relative youth of several of our patients and by the strikingly similar findings in achondroplastic dwarfs.

\section{SUMMARY}

Patients with congenital or developmental narrowing of the lumbar spinal canal suffer severely from the effects of discogenic disease because the minimal reserve space within the canal is rapidly compromised by any intruding mass. The major alterations include thickening of the laminae which overlap and are vertically orientated making it difficult to unroof the canal. Additional difficulty is provided by the presence of prominent bulbous posterior facets. The spinal canal assumes a flattened, low, triangular shape, with the maximum narrowing in the dorsoventral diameter. Small protrusions of disc tissue or osteophytes may encroach on the critical reserve space, especially in the lateral recesses, and precipitate symptoms far earlier and with greater disability than in normal individuals. Despite certain atypical features, the history and findings suggest the diagnosis of a herniated disc. Remissions are less frequent, pain soon becomes intractable and extreme. Sphincteric disturbances, sexual dysfunction, and bilateral signs of root compression may arouse suspicions of a tumour of the cauda equina or arachnoiditis. On clinical grounds alone, impressions concerning specific levels of involvement are inaccurate. Our patients presented no typical neurological syndrome. The myelographic findings, especially the discovery of a complete block, may lend support to the diagnosis of neoplasm. The evidence provided by myelography is invaluable in determining the number of vertebral levels involved.

The operation of choice consists of completely unroofing the involved areas, if necessary uncovering multiple segments. Total laminectomy is preferred to hemilaminectomy or laminotomy where bilateral changes are evident. Foramenotomy with removal of the yellow ligament, spurs, and abnormal disc tissue completes the internal decompression.

We wish to thank Dr. Leo M. Davidoff for his permission to include in this report two patients from the Neurosurgical Service of the Albert Einstein College of Medicine, Bronx, New York.

We also wish to express our gratitude to Dr. Harry Shapiro, Curator of the Department of Anthropology of the New York Museum of Natural History, for placing his collection of vertebrae at our disposal for this study.

\section{REFERENCES}

Elsberg, C. A., and Dyke, C. G. (1934). Bull. neurol. Inst. N. Y., 3, 359.

Epstein, B. S., and Epstein, J. A. (1957). Rheumatism, 13, 82.

Epstein, J. A. (1960). J. Neurosurg., 17, 991.

$\longrightarrow$, and Malis, L. I. (1955). Neurology, 5, 875.

Gill, G. G., and White, H. L. (1955). Clin. Orthop., 5, 66.

Hanraets, P. R. M. J. (1959). The Degenerative Back and its Differential Diagnosis, trans. M. E. Hollander. Elsevier, Amsterdam.

Huizinga, J., Heiden, J. A. van der, and Vinken, P. J. J. G. (1951). Proc. kon. ned. Akad. Wet., Ser. C., 55, 22. Quoted by Hanraets, P. R. M. J. (1959), and Verbiest, H. (1954 and 1955).

Jefferson, A. (1955). J. Neurol. Neurosurg. Psychiat., 18, 305.

Munro, D. (1956). New Engl. J. Med., 254, 243.

Schlesinger, E. B., and Taveras, J. M. (1953). Trans. Amer. neurol. Ass., p. 263.

Schreiber, F., and Rosenthal, H. (1952). J. Neurosurg., 9, 648.

Spillane, J. D. (1952). J. Neurol. Neurosurg. Psychiat., 15, 246.

Verbiest, H. (1954). J. Bone Jt Surg., 36-B, 230.

- (1955). Ibid., 37-B, 576.

Vogl, A., and Osborne, R. L. (1949). Arch. Neurol. Psychiat. (Chicago), 61, 644 . 